\title{
La memoria de trabajo como elemento diferencial para el Trastorno por Déficit de Atención con Hiperactividad
}

\author{
Working Memory as a differential element for Attention Deficit-Hyperactivity Disorder
}

\author{
Sara San-Nicolás ${ }^{1 *}$, loseba Iraurgi ${ }^{1}$, Ana-Berta Jara ${ }^{2}$, Miriam Azpiri ${ }^{2}$, Maite Urizar ${ }^{2}$ \\ 1 Deusto-Salud, I+D+i en Psicología Clínica y de la Salud. Facultad de Psicología y Educación. Universidad de Deusto. Bilbao, España. \\ 2 Centro de Salud Mental Infanto-Juvenil de Galdakao. Osakidetza, Servicio Vasco de Salud. Bizkaia, España.
}

\begin{abstract}
Resumen
Introducción: El creciente número de niños derivados a consultas especializadas bajo sospecha de presentar un Trastorno por Déficit de Atención con Hiperactividad (TDAH) nos lleva buscar características comunes a todos ellos que ayuden a comprender esta sobredemanda. Objetivos: Analizar las diferencias y semejanzas en Memoria de Trabajo (MT), por ser esta la función ejecutiva más vulnerable a la distracción. Método: Se analizó una muestra de 48 niños y niñas derivados desde pediatría por presunto TDAH. Se midió el Cociente Intelectual (Cl) y la MT. Resultados: El subtipo combinado era el que mostraba puntuaciones más bajas. Al margen de los objetivos propuestos, se encontraron diferencias significativas en el área de Comprensión Verbal. Discusión: Se aprecian diferencias relevantes entre los subtipos de TDAH que plantean la posibilidad de que ambos subtipos correspondan a nosologías diferentes.
\end{abstract}

Palabras clave: trastorno por déficit de atención e hiperactividad, TDAH, infancia, memoria de trabajo, funciones ejecutivas

\begin{abstract}
Introduction: The increasing number of children referred to Children and Adolescents Psychiatric Services under a suspect of showing an ADHD and the low proportion in which this diagnosis is confirmed, leads us to look for common characteristics in them all that help to find the cause of this over-demand. Objectives: To analyze the differences and similarities in Working Memory (WM) since it is the more vulnerable to distraction executive function and the one that affects the more to learning ability. Method: $A$ clinical sample of 48 children (81.3\%, males) with ages between six and 12 , derived from Pediatrics under a supposed ADHD. Intelligence Quotient (IQ) and WM in both verbal and visuo-spatial modalities were measured. Results: The were not found statistically significant differences in WM between the diverse diagnostic groups and ADHD group, although combined subtype showed the lowest outcomes in both verbal and visuo-spatial modalities of these tasks. Besides the objectives, there were found significant differences in Verbal Comprehension area (VC), being this index higher in case of ADHD group. Discussion: There were not found specific difficulties in WM between children diagnosed as ADHD regarding those described as "other disorders", though relevant differences in other areas are noticeable between ADHD subtypes. This fact suggests the possibility of considering both subtypes as independent nosologies. The convenience of further investigation to that respect through more controlled methodology is proposed.
\end{abstract}

Keywords: Attention Deficit Hyperactivity Disorder, ADHD, childhood, executive functions, working memory, differential diagnosis

\footnotetext{
*Correspondencia: s.sannicolas@deusto.es. Sara San Nicolás. DeustoSalud. Facultad de Psicología y Educación. Universidad de Deusto. Avenida de las Universidades 24. 48011. Bilbao, España. Teléfono/ fax: +34 (944) 139085 ext. 2686, +34 (666) 760678 Recibido: 15-07-11. Revisión desde: 01-07-11. Aceptado: 20-12-11
}

DOI: $10.5839 /$ rcnp.2011.0602.06 


\section{Introducción}

Actualmente, el número de demandas de asistencia clínica bajo sospecha de TDAH está aumentando considerablemente (Polanczyk, De Lima, Horta, Biederman y Rohde, 2007), si bien los diagnósticos clínicos asignados finalmente tan solo corresponden con este trastorno en un $24 \%$ de los casos (Lasa y Jorquera, 2010). Una probable razón de esta hiperdemanda es la existencia de signos y síntomas como la desatención e impulsividad en múltiples trastornos psicológicos de la infancia (Matson, Mahan, Hess y Fodstad, 2010; Rochelle, Witton y Talcott, 2009), de ahí la necesidad de establecer vías de diagnóstico diferencial.

Diversos autores han estudiado el funcionamiento ejecutivo en niños con TDAH, especialmente la Memoria de Trabajo (MT) (Etchepareborda, Paiva-Barón y Abad, 2009; López-Villalobos et al., 2010; Martín-González et al., 2008; Martinussen y Tannock, 2006; Morales y Meneses, 2003), ya que las principales dificultades que presentan estos niños son dependientes de procesos superiores, tales como la MT, la internalización del discurso y la inhibición comportamental, todos ellos componentes de las funciones ejecutivas (FE) (Barkley, 2006; Papazian, Alfonso, Luzondo y Araguez, 2009).

A este respecto, Solovieva, Quintanar y Bonilla (2003) hallaron dificultades en tareas de FE en los niños con diagnóstico de Trastorno por Déficit de Atención sin hiperactividad (TDA), lo que interpretaron como una falta de maduración de algunos factores neuropsicológicos en estos niños, lo cual justificaría la falta de éxito en el aprendizaje escolar. En estudios similares (Weber, Jourdan-Mosser y Halsband; 2007; Huang-Pollock, y Karalunas, 2010) los niños que presentaban TDAH con subtipo hiperactivo revelaban un mayor rendimiento neuropsicológico. Por otra parte, Kaufmann et al. (2010) no encontraron diferencias entre TDAH combinado y desatento en atención visoespacial y tareas de flexibilidad atencional.

Son escasos los estudios comparativos de FEs entre niños con TDAH y niños con otros trastornos, motivo por el que proponemos este estudio, siendo nuestro objetivo principal analizar las diferencias y semejanzas en MT en niños que consultan en un servicio de psiquiatría infantil por sospecha de presentar este trastorno.

\section{Método}

\section{Muestra}

Han participado 48 sujetos de entre seis y 12 años, siendo el $81,3 \%$ niños y el resto $(18,7 \%)$ niñas. La edad media es de 8,6 años, con una desviación típica de 1,5 , presentándose homogeneidad en las características socioeconómicas (clase media) y de composición familiar (en un $81 \%$ de los casos viven con ambos progenitores). Todos ellos habían sido derivados al Centro de Salud Mental Infanto-Juvenil (CSM-IJ) de Galdakao (Vizcaya, España) desde diferentes servicios de pediatría bajo sospecha de TDAH, aludida por parte de la familia o del sistema escolar, sin que hubiera una confirmación diagnóstica validada. Como criterios de inclusión se establecieron: (1) un $\mathrm{Cl}$ superior a 70 (corte para el diagnóstico de Retraso Mental según el DSM IV-TR) y (2) que los niños no presentasen ningún trastorno con implicaciones neurológicas graves o que impidiesen la administración de las pruebas.

\section{Procedimiento}

Una vez recibida en el CSM-IJ la demanda de consulta, se asignaba el menor a un profesional clínico de referencia. En una primera sesión el clínico responsable del caso informaba a los padres de las características del estudio, solicitando su participación en él. En caso de acceder a dicha colaboración, firmaban el consentimiento informado oportuno. Independientemente de su participación en el estudio, los menores eran citados para nuevas sesiones de evaluación, prosiguiendo la intervención necesaria a su caso. Las sesiones de evaluación se dividían en dos fases. Por una parte, el clínico responsable evaluaba el caso de forma independiente a través de una entrevista estructurada, sobre la que posteriormente establecía el diagnóstico. Por otra, los menores respondían a una serie de pruebas neuropsicológicas (véase instrumentos) conducidas por un grupo de evaluadores entrenados para tal fin. Para evitar sesgos producidos por el cansancio, la aplicación era fraccionada en dos sesiones, con una diferencia entre ambas de una semana. Tras la fase de evaluación, se procedía a la corrección e interpretación de las pruebas. Estos datos eran posteriormente transmitidos a los padres mediante un informe escrito y una entrevista con el profesional responsable.

Asimismo, cabe destacar que cuatro niños sobre el total de los analizados (8,3\%) se encontraban tomando metilfenidato durante el proceso de evaluación y aplicación de instrumentos.

\section{Instrumentos}

1. Escala de Inteligencia de Wechsler para Niños (WISC-IV; Wechsler, 2007). Arroja cinco puntuaciones principales: una medida de la capacidad intelectual general (CIT) y cuatro índices principales: Comprensión Verbal (CV), Razonamiento Perceptivo (RP), Memoria de Trabajo (MT) y Velocidad de Procesamiento (VP). Asimismo, incluye un índice de distractibilidad (Factor K). Una mayor puntuación en este es indicativa de un menor efecto de la distracción.

2. Figura Compleja de Rey-Osterrieth (ROCF; Rey, 1994). Sirve para evaluar las habilidades de organización perceptual, así como la memoria visoespacial y los procesos de construcción. En este estudio se utilizó para descartar que el componente verbal esté ejerciendo una influencia sobre la MT.

\section{Análisis de datos}

Los análisis estadísticos han sido realizados mediante la aplicación informática SPSS en su versión 17. Para analizar las variables clínicas y demográficas de la muestra se han utilizado los estadísticos descriptivos [media (M) y desviación típica (DT)], en el caso de variables cuantitativas, y frecuencia y porcentaje para las variables de tipo nominal o categorial.

Atendiendo al diagnóstico elaborado por el clínico de referencia se han establecido diferentes grupos de comparación. En primer lugar, y siguiendo el objetivo principal de nuestro estudio, interesa valorar la ejecución respecto a la MT entre menores con TDAH respecto de otros posibles diag- 
San-Nicolás, et al. Rev. chil. neuropsicol. 6(2): 91-98, 2011

Tabla 1. Contraste de medias (t de Student) para las variables principales (Prueba de Rey y WISC) entre en grupo TDAH y No TDAH

\begin{tabular}{|c|c|c|c|c|c|c|}
\hline Variables & Grupos & Media & D.E. & $t$ & g.l. & $\mathrm{p}$ \\
\hline \multicolumn{7}{|l|}{ Figura Compleja de Rey } \\
\hline Puntuación Copia Figura & TDAH $(n=15)$ & 79,20 & 31,67 & 0,89 & 18,18 & 0,384 \\
\hline \multirow[t]{3}{*}{ Puntuación Memoria Figura } & No TDAH $(n=33)$ & 87,00 & 17,86 & & & \\
\hline & $\operatorname{TDAH}(n=15)$ & 59,33 & 35,68 & 0,94 & 46 & 0,354 \\
\hline & No TDAH $(n=33)$ & 69,60 & 34,99 & & & \\
\hline \multicolumn{7}{|l|}{ WISC-IV } \\
\hline $\mathrm{Cl}$ Total & TDAH $(n=15)$ & 104,87 & 11,91 & $-1,56$ & 46 & 0,126 \\
\hline \multirow[t]{2}{*}{ Comprensión Verbal } & No TDAH $(n=33)$ & 99,00 & 12,17 & & & \\
\hline & TDAH $(n=15)$ & 109,20 & 12,52 & $-2,26$ & 46 & 0,028 \\
\hline \multirow[t]{2}{*}{ Razonamiento Perceptivo } & No TDAH $(n=33)$ & 100,54 & 12,17 & & & \\
\hline & TDAH $(n=15)$ & 109,00 & 14,94 & $-1,01$ & 46 & 0,320 \\
\hline \multirow[t]{2}{*}{ Memoria de Trabajo } & No TDAH $(n=33)$ & 104,70 & 13,18 & & & \\
\hline & TDAH $(n=15)$ & 96,87 & 16,77 & $-0,32$ & 46 & 0,747 \\
\hline \multirow{2}{*}{ Velocidad de Procesamiento } & No TDAH $(n=33)$ & 95,24 & 15,74 & & & \\
\hline & TDAH $(n=15)$ & 101,47 & 14,95 & $-0,78$ & 46 & 0,439 \\
\hline \multirow[t]{2}{*}{ Aritmética } & No TDAH $(n=33)$ & 98,48 & 10,89 & & & \\
\hline & TDAH $(n=15)$ & 9,20 & 3,19 & $-0,14$ & 46 & 0,892 \\
\hline \multirow[t]{2}{*}{ Dígitos } & No TDAH $(n=33)$ & 9,06 & 3,31 & & & \\
\hline & TDAH $(n=15)$ & 8,60 & 2,20 & 0,35 & 46 & 0,727 \\
\hline \multirow[t]{2}{*}{ Dígitos orden Directo } & No TDAH $(n=33)$ & 8,88 & 2,69 & & & \\
\hline & TDAH $(n=15)$ & 5,80 & 1,61 & 0,66 & 45 & 0,510 \\
\hline \multirow[t]{2}{*}{ Dígitos orden Inverso } & No TDAH $(n=32)$ & 6,12 & 1,54 & & & \\
\hline & $\operatorname{TDAH}(n=15)$ & 5,46 & 1,18 & 0,83 & 45 & 0,411 \\
\hline \multirow[t]{2}{*}{ Letras y Números } & No TDAH $(n=32)$ & 5,87 & 1,71 & & & \\
\hline & TDAH $(n=15)$ & 9,06 & 3,61 & $-0,08$ & 46 & 0,939 \\
\hline \multirow[t]{3}{*}{ Factor de Distractibilidad (K) } & No TDAH $(n=33)$ & 8,96 & 4,20 & & & \\
\hline & $\operatorname{TDAH}(n=15)$ & 8,95 & 2,26 & 0,18 & 46 & 0,859 \\
\hline & No TDAH $(n=33)$ & 9,09 & 2,50 & & & \\
\hline
\end{tabular}

nósticos ('grupo No-TDAH'). Para comparar los resultados alcanzados por ambos grupos respecto a la MT y otras variables de interés se han utilizado pruebas $t$ de Student para grupos independientes, aplicando la prueba de corrección en el caso de que la prueba de Levene indicase heterogeneidad de varianzas.

No obstante, dada la potencial diversidad de trastornos diagnosticados y el posible comportamiento diferencial de los mismos respecto a la MT, se ha procedido a realizar análisis de $\mathrm{K}$ grupos a través de la prueba de KruskalWallis. En aquellas situaciones en las que se ha hallado significación estadística $(p<0,05)$ y/o tendencial $(p 0,10)$, se han realizado comparaciones post-hoc entre los pares de clasificaciones diagnósticas posibles basados en la prueba de Mann-Whitney.

\section{Resultados}

\section{Distribución diagnóstica}

Los 48 menores participantes acudieron al servicio de salud mental derivados bajo sospecha de TDAH, pero el diagnóstico final validado no correspondía en todos los casos con este trastorno. Los diagnósticos resultantes se integran en seis grupos: Trastornos de las Emociones (13 casos, 27\%); Trastorno por Déficit de Atención subtipo desatento (TDAH-D; ocho casos $16,6 \%)$, Trastorno por Déficit de Atención subtipo combinado (TDAH-C; siete casos, 14,6\%), Trastorno Neurótico (siete casos, $14,6 \%$ ) y Trastorno Disocial (cuatro casos, $8,3 \%$ ). Asimismo, un conjunto de trastornos de baja frecuencia han sido enmarcados bajo el epígrafe de "Otros Trastornos" (nueve casos, 18,7\%), entre los que destacan el Trastorno del Aprendizaje $(n=3)$, Trastorno del Desarrollo Psicológico $(n=3)$, Trastorno Adap- tativo $(n=1)$, Trastornos por Estrés Postraumático $(n=1)$ y Trastorno Generalizado del Desarrollo $(n=1)$.

Atendiendo a las características de identificación de nuestro estudio, quedarían subsumidos bajo el epígrafe TDAH los casos enmarcados bajo los diagnósticos de TDAH desatento y combinado, lo que supone un conjunto de 15 casos sobre el total de la muestra (31,3\%).

\section{Memoria de Trabajo}

El análisis de la varianza mediante la prueba $t$ (ver tabla 1) muestra que no existen diferencias estadísticamente significativas entre los grupos TDAH y no TDAH en el nivel de Memoria de Trabajo ni en su vertiente espacial (ROCF) ni verbal (Índice MT del WISC). Sin embargo, sí se encuentran diferencias en el índice de Comprensión Verbal (CV) $(t=-2,26 ; p=0,028)$, siendo el grupo TDAH el que mejor ejecución presenta ( $\mathrm{M}_{\mathrm{TDAH}}=109,20$ vs $\mathrm{M}_{\text {No-TDAH }}=100,54$ ).

En la tabla 2 se muestran los análisis realizados mediante la prueba de Kruskal-Wallis, desglosados por categorías diagnósticas, con el fin de detectar si la heterogeneidad del grupo no-TDAH podría estar ejerciendo un efecto de confusión sobre los resultados. Se hallaron diferencias significativas en el subtest dígitos (D) $\left(\mathrm{K}-\mathrm{W} \chi_{(5)}^{2}=11,59 ; \mathrm{p}=\right.$ $0,041)$, mostrándose esta diferencia de forma más especifica en el caso de la prueba de dígitos en orden directo (Dd) $\left(\mathrm{K}-\mathrm{W} \chi_{(5)}^{2}=\right.$ $12,48 ; p=0,029)$. Además, destacan diferencias tendenciales en la ROCF en su fase de Memoria $\left(K-W \chi_{(5)}^{2}=9,17 ; p=0,102\right)$ y el índice CV $\left(\mathrm{K}-\mathrm{W} \chi_{(5)}^{2}=8,83 ; \mathrm{p}=0,116\right)$.

En estos cuatro casos se llevaron a cabo un total de 60 pruebas de Mann-Whitney (15 comparaciones para cada índice 
Tabla 2. Contraste de medias para las variables de inteligencia (Prueba de Rey y WISC). Análisis de Varianza (continúa en la página siguiente).

\begin{tabular}{|c|c|c|c|c|c|c|}
\hline Variables & Grupos & Media & D.E. & $x^{2}$ & g.l. & $\mathrm{p}$ \\
\hline \multicolumn{7}{|l|}{ Figura Compleja de Rey } \\
\hline \multirow[t]{6}{*}{ Puntuación Copia Figura } & TDAH-D $(n=8)$ & 86,25 & 25,68 & 2,70 & 5 & 0,745 \\
\hline & TDAH-C $(n=7)$ & 71,14 & 37,80 & & & \\
\hline & Trast. emociones $(n=13)$ & 91,53 & 7,63 & & & \\
\hline & Trast. neuróticos $(n=7)$ & 78,28 & 25,33 & & & \\
\hline & Trast. disociales $(n=4)$ & 79,50 & 33,27 & & & \\
\hline & Otros $(n=9)$ & 90,55 & 12,25 & & & \\
\hline \multirow[t]{6}{*}{ Puntuación Memoria Figura } & TDAH-D $(n=8)$ & 70,75 & 35,57 & 9,17 & 5 & 0,102 \\
\hline & TDAH-C $(n=7)$ & 46,28 & 33,48 & & & \\
\hline & Trast. emociones $(n=13)$ & 81,53 & 21,40 & & & \\
\hline & Trast. neuróticos $(n=7)$ & 54,71 & 40,35 & & & \\
\hline & Trast. disociales $(n=4)$ & 32,50 & 45,01 & & & \\
\hline & Otros (n=9) & 80,44 & 31,35 & & & \\
\hline \multicolumn{7}{|l|}{ WISC-IV } \\
\hline \multirow[t]{6}{*}{ Cl Total } & TDAH-D $(n=8)$ & 108,00 & 12,93 & 4,21 & 5 & 0,519 \\
\hline & TDAH-C $(n=7)$ & 101,29 & 10,38 & & & \\
\hline & Trast. emociones $(n=13)$ & 101,92 & 12,36 & & & \\
\hline & Trast. neuróticos $(n=7)$ & 100 & 13,16 & & & \\
\hline & Trast. disociales $(n=4)$ & 96,25 & 6,55 & & & \\
\hline & Otros $(n=9)$ & 95,22 & 13,51 & & & \\
\hline \multirow[t]{6}{*}{ Comprensión Verbal } & TDAH-D $(n=8)$ & 109,00 & 14,90 & 8,83 & 5 & 0,116 \\
\hline & TDAH-C $(n=7)$ & 109,42 & 10,30 & & & \\
\hline & Trast. emociones ( $n=13$ ) & 103 & 11,53 & & & \\
\hline & Trast. neuróticos $(n=7)$ & 101,42 & 15,02 & & & \\
\hline & Trast. disociales $(n=4)$ & 90 & 6,63 & & & \\
\hline & Otros $(n=9)$ & 101 & 11,88 & & & \\
\hline \multirow[t]{6}{*}{ Razonamiento Perceptivo } & TDAH-D $(n=8)$ & 111,63 & 15,06 & 2,74 & 5 & 0,740 \\
\hline & TDAH-C $(n=7)$ & 106 & 15,37 & & & \\
\hline & Trast. emociones $(n=13)$ & 107,69 & 11,41 & & & \\
\hline & Trast. neuróticos $(n=7)$ & 100,29 & 13,45 & & & \\
\hline & Trast. disociales $(n=4)$ & 107,50 & 6,75 & & & \\
\hline & Otros $(n=9)$ & 102,56 & 17,48 & & & \\
\hline \multirow[t]{6}{*}{ Memoria de Trabajo } & TDAH-D $(n=8)$ & 104,75 & 19,91 & 5,39 & 5 & 0,370 \\
\hline & TDAH-C $(n=7)$ & 87,86 & 4,01 & & & \\
\hline & Trast. emociones $(n=13)$ & 95,31 & 15,62 & & & \\
\hline & Trast. neuróticos $(n=7)$ & 99,71 & 19,36 & & & \\
\hline & Trast. disociales $(n=4)$ & 97,50 & 15,84 & & & \\
\hline & Otros $(n=9)$ & 90,67 & 14,35 & & & \\
\hline \multirow[t]{6}{*}{ Velocidad de Procesamiento } & TDAH-D $(n=8)$ & 104 & 16,77 & 3,90 & 5 & 0,564 \\
\hline & TDAH-C $(n=7)$ & 98,57 & 13,52 & & & \\
\hline & Trast. emociones $(n=13)$ & 100,08 & 10,65 & & & \\
\hline & Trast. neuróticos $(n=7)$ & 101,43 & 6,85 & & & \\
\hline & Trast. disociales $(n=4)$ & 99,50 & 13,52 & & & \\
\hline & Otros $(n=9)$ & 93,44 & 12,64 & & & \\
\hline \multirow[t]{6}{*}{ Aritmética } & TDAH-D $(n=8)$ & 9,63 & 4,06 & 0,67 & 5 & 0,984 \\
\hline & TDAH-C $(n=7)$ & 8,71 & 1,97 & & & \\
\hline & Trast. emociones ( $n=13)$ & 9,46 & 3,71 & & & \\
\hline & Trast. neuróticos $(n=7)$ & 9,14 & 2,61 & & & \\
\hline & Trast. disociales $(n=4)$ & 9,50 & 2,64 & & & \\
\hline & Otros $(n=9)$ & 8,22 & 3,80 & & & \\
\hline \multirow[t]{6}{*}{ Dígitos } & TDAH-D $(n=8)$ & 10 & 1,60 & 11,59 & 5 & 0,041 \\
\hline & TDAH-C $(n=7)$ & 7 & 1,63 & & & \\
\hline & Trast. emociones $(n=13)$ & 9,07 & 2,39 & & & \\
\hline & Trast. neuróticos $(n=7)$ & 9 & 2,94 & & & \\
\hline & Trast. disociales $(n=4)$ & 11 & 3,55 & & & \\
\hline & Otros $(n=9)$ & 7,55 & 2,18 & & & \\
\hline Dígitos orden Directo & TDAH-D $(n=8)$ & 6,87 & 0,99 & 12,48 & 5 & 0,029 \\
\hline & TDAH-C $(n=7)$ & 4,57 & 1,27 & & & \\
\hline & Trast. emociones $(n=13)$ & 6 & 1,41 & & & \\
\hline & Trast. neuróticos $(n=7)$ & 6,57 & 1,51 & & & \\
\hline & Trast. disociales $(n=4)$ & 7 & 1,82 & & & \\
\hline & Otros $(n=9)$ & 5,55 & 1,58 & & & \\
\hline Dígitos orden Inverso & TDAH-D $(n=8)$ & 5,75 & 1,16 & 6,72 & 5 & 0,242 \\
\hline & TDAH-C $(n=7)$ & 5,14 & 1,12 & & & \\
\hline & Trast. emociones $(n=13)$ & 6,83 & 1,89 & & & \\
\hline & Trast. neuróticos $(n=7)$ & 5,28 & 1,49 & & & \\
\hline & Trast. disociales $(n=4)$ & 5,25 & 1,25 & & & \\
\hline & Otros $(n=9)$ & 5,33 & 1,41 & & & \\
\hline
\end{tabular}

D.E.: Desviación estándar ; g.I. Grados de libertad 
San-Nicolás, et al. Rev. chil. neuropsicol. 6(2): 91-98, 2011

Tabla 2. Contraste de medias para las variables de inteligencia (Prueba de Rey y WISC). Análisis de Varianza (Continuación)

\begin{tabular}{|c|c|c|c|c|c|c|}
\hline Variables & Grupos & Media & D.E. & $\mathrm{X} 2$ & g.l. & $\mathrm{p}$ \\
\hline \multicolumn{7}{|l|}{ WISC-IV } \\
\hline \multirow[t]{6}{*}{ Letras y Números } & TDAH-D $(n=8)$ & 10,25 & 4,13 & 7,02 & 5 & 0,219 \\
\hline & TDAH-C $(n=7)$ & 7,71 & 2,56 & & & \\
\hline & Trast. emociones $(n=13)$ & 9,92 & 3,45 & & & \\
\hline & Trast. neuróticos $(n=7)$ & 10,14 & 3,53 & & & \\
\hline & Trast. disociales $(n=4)$ & 3,75 & 6,65 & & & \\
\hline & Otros $(n=9)$ & 9 & 3,27 & & & \\
\hline \multirow[t]{6}{*}{ Factor de Distractibilidad (K) } & TDAH-D $(n=8)$ & 9,95 & 2,55 & 5,01 & 5 & 0,414 \\
\hline & TDAH-C $(n=7)$ & 7,80 & 1,23 & & & \\
\hline & Trast. emociones $(n=13)$ & 9,48 & 2,67 & & & \\
\hline & Trast. neuróticos $(n=7)$ & 9,42 & 2,74 & & & \\
\hline & Trast. disociales $(n=4)$ & 9,08 & 1,96 & & & \\
\hline & Otros $(n=9)$ & 8,25 & 2,47 & & & \\
\hline
\end{tabular}

D.E.: Desviación estándar ; g.l. Grados de libertad

Tabla 3. Contraste de medias para las variables principales (Prueba de Rey y WISC) entre grupo bajo tratatamiento con metilfenidato vs. grupo sin tratamiento. Análisis de Varianza

\begin{tabular}{|c|c|c|c|c|c|c|}
\hline Variable & Grupos & Media & D.E. & $F$ & g.l. & $\mathrm{p}$ \\
\hline \multicolumn{7}{|l|}{ Figura Compleja de Rey } \\
\hline Puntuación Copia Figura & $\operatorname{MTF}(n=4)$ & 92,00 & 9,05 & 0,45 & 46 & 0,505 \\
\hline \multirow[t]{3}{*}{ Puntuación Memoria Figura } & No MTF $(n=44)$ & 83,88 & 23,81 & & & \\
\hline & $\operatorname{MTF}(n=4)$ & 72,00 & 34,97 & 0,11 & 46 & 0,743 \\
\hline & No $\operatorname{MTF}(n=44)$ & 65,88 & 42,29 & & & \\
\hline \multicolumn{7}{|l|}{ WISC-IV } \\
\hline \multirow[t]{2}{*}{ Cl Total } & $\operatorname{MTF}(n=4)$ & 103,25 & 17,29 & 0,16 & 46 & 0,685 \\
\hline & No $\operatorname{MTF}(n=44)$ & 100,61 & 11,97 & & & \\
\hline \multirow[t]{2}{*}{ Comprensión Verbal } & $\operatorname{MTF}(n=4)$ & 100,25 & 18,39 & 0,23 & 46 & 0,630 \\
\hline & No $\operatorname{MTF}(n=44)$ & 103,52 & 12,44 & & & \\
\hline \multirow[t]{2}{*}{ Razonamiento Perceptivo } & $\operatorname{MTF}(n=4)$ & 105,75 & 16,31 & 0,02 & 46 & 0,965 \\
\hline & No $\operatorname{MTF}(n=44)$ & 106,07 & 13,70 & & & \\
\hline \multirow[t]{2}{*}{ Memoria de Trabajo } & $\operatorname{MTF}(n=4)$ & 99,25 & 15,96 & 0,21 & 46 & 0,651 \\
\hline & No $\operatorname{MTF}(n=44)$ & 95,43 & 16,04 & & & \\
\hline \multirow[t]{2}{*}{ Velocidad de Procesamiento } & $\operatorname{MTF}(n=4)$ & 109,00 & 16,79 & 2,78 & 46 & 0,102 \\
\hline & No $\operatorname{MTF}(n=44)$ & 98,55 & 11,58 & & & \\
\hline \multirow[t]{2}{*}{ Aritmética } & $\operatorname{MTF}(n=4)$ & 7,50 & 1,91 & 1,07 & 46 & 0,307 \\
\hline & No $\operatorname{MTF}(n=44)$ & 9,25 & 3,31 & & & \\
\hline \multirow[t]{2}{*}{ Dígitos } & $\operatorname{MTF}(n=4)$ & 10,25 & 1,26 & 1,47 & 46 & 0,232 \\
\hline & No $\operatorname{MTF}(n=44)$ & 8,66 & 2,58 & & & \\
\hline \multirow[t]{2}{*}{ Dígitos orden Directo } & $\operatorname{MTF}(n=4)$ & 6,75 & 1,50 & 0,96 & 45 & 0,332 \\
\hline & No $\operatorname{MTF}(n=44)$ & 5,95 & 1,55 & & & \\
\hline \multirow[t]{2}{*}{ Dígitos orden Inverso } & $\operatorname{MTF}(n=4)$ & 5,50 & 1,91 & 0,10 & 45 & 0,748 \\
\hline & No $\operatorname{MTF}(n=44)$ & 5,76 & 1,55 & & & \\
\hline \multirow[t]{2}{*}{ Letras y Números } & $\operatorname{MTF}(n=4)$ & 9,50 & 4,65 & 0,07 & 46 & 0,797 \\
\hline & No $\operatorname{MTF}(n=44)$ & 8,95 & 3,99 & & & \\
\hline \multirow[t]{2}{*}{ Factor de Distractibilidad (K) } & $\operatorname{MTF}(n=4)$ & 9,08 & 2,17 & 0,01 & 46 & 0,976 \\
\hline & No $\operatorname{MTF}(n=44)$ & 9,04 & 2,45 & & & \\
\hline
\end{tabular}

MTF: Metilfenidato; D.E.: Desviación estándar ; g.I. Grados de libertad

valorado), de las cuales 10 han alcanzado significación estadística $(p<0,05)$. Atendiendo a la prueba de la ROCF, fase de Memoria, se han encontrado diferencias estadísticamente significativas entre el grupo de menores con TDAH-C $(M=46,28)$ vs. los Trastornos de las Emociones $(M=81,53)$ y Otros Trastornos ( $M=80,44)$; pruebas de Mann-Whitney de $z=-2,25$ $(p=0,024)$ y $z=-1,94(p=0,052)$, respectivamente. En cuanto al índice de $\mathrm{CV}$, las diferencias halladas se expresan entre el grupo de Trastornos Disociales $(M=90,00)$ respecto a TDAH-D (M= $109,00)$ y TDA-C $(M=109,42)$; pruebas de Mann-Whitney de $z=-$ $2,04(p=0,041)$ y $z=-2,46(p=0,014)$, respectivamente. Por su parte, el caso de $D$ se han encontrado diferencias significativas entre los grupos TDAH-D $(M=10,00)$ vs. TDAH-C $(M=7,00 ; z=-$ $2,62 ; p=0,007)$ y Otros Trastornos ( $M=7,55 ; z=-2,24 ; p=0,025)$, $y$ entre el grupo TDAH-C vs. Trastornos Disociales $(M=11,00 ; z=$
$-2,18 ; p=0,029)$. Finalmente, y derivado de la prueba anterior, en concreto el caso de Dígitos orden directo (Dd), casi con el mismo patrón de diferencias que en el caso anterior: TDAH-D $(M=6,87)$ vs. TDAH-C $(M=4,57 ; z=-2,82 ; p=0,005)$ y Otros Trastornos $(M=5,55 ; z=-2,02 ; p=0,043)$, y entre el grupo TDAHC vs. Trastornos Neuróticos ( $M=6,57 ; z=-2,62 ; p=0,009)$.

\section{Efectos del metilfenidato}

La prevalencia de casos tratados con metilfenidato durante el proceso de evaluación fue bajo; cuatro casos, que supone un $8,3 \%$ de total de participantes. Se valoró la posible interferencia de la medicación en la respuesta a los instrumentos, comparando el rendimiento de los menores sujetos a tratamiento con metilfenidato respecto de los no tratados. Los 
resultados (ver tabla 3), basados en la prueba de BrownForsythe, han resultado en todos los casos estadísticamente no significativos.

\section{Discusión}

El objetivo de este estudio ha consistido en hallar elementos comunes y/o diferenciales en niños consultantes por TDAH. Para ello nos hemos centrado en el área de la memoria de trabajo (MT), comúnmente afectada por la distractibilidad (Baddeley, 1999). Desde esta premisa, se ha tratado de determinar si las dificultades a este nivel son específicas del TDAH o se encuentran también en otros trastornos infantiles, justificando así la sobredemanda de consultas por motivos de TDAH (Cáceres, 2009; Kutcher et al., 2004; López et al., 2004; Polanczyk et al., 2007) cuando, finalmente, el número de niños diagnosticados por este trastorno no resulta tan elevado (Lasa y Jorquera, 2010).

La distribución diagnóstica presentada anteriormente se ha encontrado en estudios realizados sobre la misma área geográfica (Lasa y Jorquera, 2010), evidenciando una sobre etiquetación de manifestaciones infantiles bajo el epígrafe TDAH, quizá atribuyéndolo a la presencia de signos comportamentales (inquietud, impulsividad, distractibilidad,...) comunes a otras trastornos y que confunden el diagnóstico entre los no especialistas.

Se ha analizado el posible efecto del metilfenidato en cuatro niños que lo estaban tomando durante la realización del proceso de evaluación y aplicación de pruebas de rendimiento en $\mathrm{MT}$, respecto al rendimiento del resto de participantes que no lo tenían prescrito. Los resultados han mostrado ser estadísticamente no significativos, lo cual apuntaría a considerar que el efecto de una mejora de rendimiento atribuido al fármaco no resulta apreciable. Hay que hacer notar, como más tarde se argumentará en la limitaciones del estudio, que la baja prevalencia de casos tratados con metilfenidato puede influir en la potencia de la prueba estadística aplicada. Así, por ejemplo, en dos de las comparaciones realizadas, las referidas a la copia de la figura de Rey y la velocidad de procesamiento del WISC, las diferencias son mayores de 8 puntos, que, en el caso de haberse dispuesto de una muestra mayor, podría haber resultado estadísticamente significativo. No obstante, de las 13 comparaciones realizadas, tan solo las dos aludidas han mostrado un efecto apreciable, siendo en el resto prácticamente equivalentes. Por esta razón, se asume que en la muestra estudiada el efecto del metilfenidato es poco apreciable, considerando equivalencia entre los resultados de los niños tratados respecto de los que no lo son y analizando el total de casos como un conjunto.

Siguiendo con el objetivo principal del estudio, se ha pretendido discernir si el rendimiento en MT ofrece diferencias entre niños/as diagnosticados de TDAH respecto de los diagnosticados bajo otras nosologías. Los resultados iniciales han mostrado que no existen diferencias estadísticamente significativas entre ambos grupos diagnósticos, situándose los valores de rendimiento dentro de los límites normativos (Wechsler, 2007). Estos datos son consistentes con los obtenidos por Kerns, Mclnerney y Wilde (2001) y Mariani y Barkley (1997), aunque contrastan con lo postulado por otros autores (Mclnnes, Humphries, Hogg-Johnson y Tannock, 2003).
A este respecto, cabe advertir que no se ha contado con un grupo control de población no clínica, de modo que no podemos afirmar que el rendimiento en MT de los grupos evaluados sea equivalente al de este. No obstante, nuestro interés se ha circunscrito a valorar la existencia de factores comunes en las manifestaciones y/o trastornos infantiles que puedan confundir la atribución diagnóstica. Además, dado que las funciones ejecutivas se hallan en desarrollo durante las edades contempladas en este estudio, no podríamos hablar de déficits en sí mismos, sino de un enlentecimiento o inmadurez en su desarrollo (Bowers et al., 1992; Solovieva et al., 2003; Stelzer, Cervigni y Martino, 2010).

Si bien el objetivo del estudio ha sido constatar los elementos comunes entre niños/as diagnosticados de TDAH vs. otros trastornos, tal agrupación puede llegar a ser grosera, puesto que el grupo TDAH puede diferenciarse en función de la presencia o ausencia de hiperactividad y los trastornos No-TDAH pueden comprender cuadros muy heterogéneos. Por esta razón, se ha realizado un análisis secundario de los datos, lo que, dada la limitación de efectivos, ha conllevado una pérdida de potencia estadística y un mayor riesgo de cometer un error de tipo II. No obstante, nuestro interés ha radicado en explorar las posibles diferencias con entidad clínica o que sean reflejo de una diferencia teóricamente relevante (Iraurgi, 2009).

Siguiendo este procedimiento, se han observado diferencias tendenciales entre los distintos grupos diagnósticos en la fase de memoria de la ROCF y en el índice de Comprensión Verbal (CV); y diferencias estadísticamente significativas en las pruebas de Dígitos del WISC (general y orden directo). Análisis subsiguientes entre pares diagnósticos han ofrecido interesantes diferencias en los patrones de rendimiento en estos índices. Excepto en el índice de CV, el TDAH-C es el que peor rendimiento ha presentado en los índices señalados. Asimismo, se han apreciado diferencias en MT entre ambos subtipos de TDAH que, si bien no han obtenido una significación estadística, sí presentan una apreciable significación práctica. Nos referimos al hecho de que el grupo TDAH-C haya obtenido resultados más bajos en la fase de memoria de la ROCF $(M=46,28)$ que el grupo TDAH-D $(M=70,75)$. Este resultado es además importante por presentar el subtipo combinado una puntuación centil inferior a la esperada, lo que sugiere dificultades específicas en la reproducción visoespacial a través del recuerdo. Cabe destacar, asimismo, la existencia de una situación semejante en el caso del índice de MT del WISC, en el que la diferencia de medias entre ambos es también relevante ( $M_{\text {TDAH-D }}=104,75$ vs $M_{\text {TDAH-C }}=87,86$ ). Estas dificultades, tanto en el nivel verbal (WISC) como viso-espacial (ROCF), son consistentes con lo dispuesto recientemente por Kaufmann et al. (2010), aunque contrastan con otros estudios (Weber, Jourdan-Mosser y Halsband, 2007 y Huang-Pollock, y Karalunas, 2010).

Ahora bien, analizadas con detalle las puntuaciones alcanzadas por los diferentes grupos en la fase de memoria de la ROCF vs. las tareas de MT o dígitos del WISC, observamos cómo en el primer caso la puntuación más baja no es la del grupo TDAH-C ( $M=46,28)$, sino la de Trastornos Disociales ( $M=32,50)$, mientras que en las pruebas de MT y Dígitos el rendimiento alcanzado por este último grupo es mayor que el de TDAH-C $(97,50$ vs. 87,86 y 11 vs. 7 , respectivamente para cada grupo y prueba). Es decir, el grupo disocial presenta el peor rendimiento 
en un tipo de pruebas y posteriormente un rendimiento promedio en el resto, mientras que el grupo TDAH-C muestra un patrón más estable a través de las diferentes pruebas. La razón de esta disparidad se halla en la disposición actitudinal de los niños diagnosticados de Trastorno Disocial, quienes se negaban a continuar con la ejecución de la prueba de memoria del ROCF. Introducimos aquí este detalle porque consideramos que es un elemento importante en el diagnóstico clínico que, en muchas ocasiones, no se tiene en cuenta en los estudios de valoración del rendimiento de las funciones ejecutivas con fines de investigación, incurriéndose así en la falta de control de un sesgo importante y limitando, por consiguiente, el sentido de los resultados.

Otras limitaciones de nuestro estudio se circunscriben al bajo número de efectivos con los que hemos contado y a la falta de un grupo control. Las consecuencias de operar con muestras pequeñas es por todos conocido (baja potencia estadística y alto error de medición, subgrupos poco representados, etc.), pero en muchas ocasiones son los límites que nos impone trabajar con muestras clínicas. Por otro lado, la falta de comparaciones normativas ofrecidas por un grupo control, limita la interpretación y generalización de los resultados. Por todo ello, debemos tener cautela a la hora de valorar los resultados de este estudio. No obstante, estos hallazgos plantean nuevas líneas de investigación. El hecho de que las diferencias se encuentren entre los subtipos de TDAH y no entre los grupos TDAH y No-TDAH, apoya la hipótesis planteada por algunos autores (Barkley, 2001; Milich, Balentine y Lynam, 2001) que propone considerar las tipologías de TDAH como entidades diagnósticas independientes. A este respecto, intensificar los estudios sobre estas en el nivel de la MT aparece como una línea de investigación factible.

Por otro lado, se han observado diferencias en el índice de CV del WISC, evidenciando un mayor rendimiento en los diagnósticos de TDAH (en torno a los 109 puntos) respecto de otros diagnósticos. En la revisión bibliográfica efectuada no hemos encontrado evidencias que apoyen $u$ objeten estos hallazgos. A modo de hipótesis que explique nuestros resultados, tal vez la falta de inhibición propia de estos niños conduzca a una conducta prosocial y, por tanto, a un mayor desarrollo de las aptitudes comunicativas, aunque sería necesario cotejarlo con una muestra control normativa. Otra posible hipótesis es que los mecanismos implicados en las habilidades verbales difieran de los implicados en la MT. A este respecto, Baddeley (1999) afirma que el aprendizaje de las reglas gramaticales de la lengua materna requiere de la participación de la memoria implícita.

Teniendo en cuenta tanto la literatura revisada como los datos obtenidos en este estudio, no parece que los niños con TDAH tengan mayores dificultades que aquellos que presentan otros trastornos, encontrándonos así ante una injustificada alarma social con graves repercusiones sobre el funcionamiento de estos niños, debido a la falsa expectativa negativa generada hacia ellos por parte de su entorno más cercano. Por lo anterior, resulta necesario y conveniente continuar la investigación iniciada respecto de las manifestaciones y características del TDAH, tanto para conocer mejor sus aspectos idiosincráticos y cómo manejarlos de manera más exitosa, como para contener las alarmas sociales que, en ocasiones, dificultan un abordaje adecuado del trastorno.

\section{Agradecimientos}

Este estudio ha sido parcialmente financiado por una beca concedida por la Fundación Jesús de Gangoiti Barrera a la autora principal.

\section{Referencias}

Baddeley, A. (1999). Memoria humana: teoría y práctica. Madrid: Mc Graw-Hill. Barkley, R.A. (2001). The inattentive type of ADHD as a distinct disorder: What remains to be done? Clinical Psychology: Science and Practice, 8(4), 489-501.

Barkley, R.A. (2006). ADHD: A handbook for diagnosis and treatment, $3^{\mathrm{a}} \mathrm{Ed}$. New York: The Guilford Press.

Bowers, T.G., Risser, M.G., Suchanec, J.F., Tinker, D.E., Ramer, J.C y Domoto, M. (1992). A developmental index using the Wechsler Intelligence Scale for Children: Implications for the diagnosis and nature of ADHD. Journal of Learning Disabilities, 25(3), 179-185.

Cáceres-Carrasco, J. (2009). Trastorno por déficit de atención /hiperactividad ¿Existe acuerdo en el diagnóstico? Cuadernos de Medicina Psicosomática y Medicina de Enlace, 92, 26-34.

Etchepareborda, M.C., Paiva-Barón, H. y Abad, L. (2009). Ventajas de las baterías de evaluación neuropsicológica en el trastorno por déficit de atención/hiperactividad. Revista de Neurología, 48 (supl. 2), 89-93.

Huang-Pollock, C.L. y Karalunas, S.L. (2010). Working memory demands impair skill acquisition in children with ADHD. Journal of Abnormal Psychology, 119(1), 174-185.

Iraurgi, I. (2009). Evaluación de resultados clínicos I: entre la significación estadística y la relevancia clínica. Norte de Salud Mental, 33, 94-108.

Kaufmann, L., Zieren, N., Zotter, S., Karall, D., Scholl- Bürgi, S., Haberlandt, E. y Fimm, B. (2010). Predictive validity of attentional functions in differentiating children with and without ADHD: a componential analysis. Developmental Medicine and Child Neurology, 52(4), 371-378.

Kerns, K.A., Mclnerney, R.J. y Wilde, N.J. (2001). Time reproduction, working memory and behavioral inhibition in children with ADHD. Child Neuropsychology, 7(1), 21-32.

Kutcher, S., Aman, M., Brooks, S.J., Buitelaar, J. Van Daalen, E., Fegert, J. et al. (2004). Declaración de consenso internacional sobre el trastorno de déficit de atención e hiperactividad y los trastornos de conducta disruptiva: implicaciones clínicas y sugerencias de tratamiento. European Neuropsychopharmacology, 14(1), 11-28.

Lasa, A. y Jorquera, C. (2010). Evaluación de la situación asistencial y recomendaciones terapéuticas en el trastorno por déficit de atención e hiperactividad. Informes de evaluación de tecnologías sanitarias. Osteba (2007-2009).

López, J.A., Serrano, I., Delgado, J., Ruiz F., García M.U. y Sánchez, M.I. (2004). Trastorno por déficit de atención con hiperactividad: una alteración psicopatológica con impacto multidimensional. Anales de Psiquiatría, 20(5), 205-210.

López-Villalobos, J.A., Serrano-Pintado, I., Andrés-De-Llano, J.M., Sánchez-Mateos, J.D., Alberola-López, S. y Sánchez-Azón, M.I. (2010). Utilidad del test de Stroop en el trastorno por déficit de atención/hiperactividad. Revista de Neurología, 50(6), 333-340.

Mariani, M.A. y Barkley, R.A. (1997). Neuropsychological and academic functioning in preschool boys with attention deficit hyperactivity disorder. Developmental Neuropsychology, 13(1), 111-129.

Martín-González, R., González-Pérez, P.A., Izquierdo-Hernández, M., HernándezExpósito, S., Alonso-Rodríguez, M.A., Quintero-Fuentes, I. y Rubio-Morell, B. (2008). Evaluación neuropsicológica de la memoria en el trastorno por déficit de atención/hiperactividad: papel de las funciones ejecutivas. Revista de Neurología, 47(5), 225-230.

Martinussen, R. y Tannock, R. (2006). Working Memory impairments in children with Attention-Deficit Hyperactivity Disorder with and without comorbid Language Learning Disorders. Journal of Clinical \& Experimental Neuropsychology, 28(7), 1073-1094.

Matson, J.L., Mahan, S., Hess, J.A. y Fodstad, J.C. (2010). Effect of developmental quotient on symptoms of inattention and impulsivity among toddlers with Autism Spectrum Disorders. Research in Developmental Disabilities, 31(2), 464-469.

Mclnnes, A., Humphries, T., Hogg-Johnson, S. y Tannock, R. (2003). Listening Comprehension and Working Memory Are Impaired in Attention-Deficit Hyperactivity Disorder Irrespective of Language Impairment. Journal of Abnormal Child Psychology, 31(4), 427-445.

Milich, R., Balentine, A.C. y Lynam, D.R. (2001). ADHD combined type and ADHD predominantly inattentive type are distinct and unrelated disorders. Clinical Psychology: Science and Practice, 8(4), 463-488. 
Morales, G. y Meneses, S. (2003). Evaluación de procesos atencionales y funciones ejecutivas en niños con trastorno de la atención con hiperactividad. Revista Neuropsicología, Neuropsiquiatría y Neurociencias, 5, 138-158.

Polanczyk, G., de Lima, M.S., Horta, B.L., Biederman, J. y Rohde, L.A. (2007). The worldwide prevalence of ADHD: a systematic review and metaregression analysis. American Journal of Psychiatry, 164 (6), 942-948.

Papazian O, Alfonso I, Luzondo RJ, Araguez N. (2009). Entrenamiento de la función ejecutiva en preescolares con trastorno por déficit de atención/hiperactividad combinado: estudio prospectivo, controlado y aleatorizado. Revista de Neurología, 48 (Supl.2), 119-122.

Rey, A. (1994). Test de copia de una figura compleja. Madrid: Tea Ediciones.

Rochelle, K.S.H., Witton, C. y Talcott, J. (2009). Symptoms of hyperactivity and inattention can mediate deficits of postural stability in developmental dyslexia. Experimental Brain Research, 192(4), 627-663.

Solovieva, Y., Quintanar Rojas, L. y Bonilla Sánchez, M.R. (2003). Análisis de las funciones ejecutivas en niños con déficit de atención. Revista Española de Neuropsicología, 5(2), 163-176.

Stelzer, F., Cervigni, M.A. y Martino, P. (2010). Bases neurales del desarrollo de las funciones ejecutivas durante la infancia y adolescencia. Una revisión. Revista Chilena de Neuropsicología, 5(3), 176-184.

Weber, P., Jourdan-Moser, S. y Halsband, U. (2007). Differences between subtypes of children with ADHD and simple attentional difficulties during an initial assessment. Acta Pædiatrica, 96, 1511-1517.

Wechsler, D. (2007). WISC-IV Escala de Inteligencia de Wechsler para Niños-IV. Manual técnico y de interpretación. 2a ed. Madrid: TEA Ediciones. (Trabajo original publicado en 2003). 